\title{
Three-body charmed baryon decays with SU(3) flavor symmetry
}

\author{
C. Q. Geng, ${ }^{1,2,3}$ Y. K. Hsiao, ${ }^{1}$ Chia-Wei Liu, ${ }^{2}$ and Tien-Hsueh Tsai ${ }^{2}$ \\ ${ }^{1}$ School of Physics and Information Engineering, Shanxi Normal University, Linfen 041004 \\ ${ }^{2}$ Department of Physics, National Tsing Hua University, Hsinchu 300 \\ ${ }^{3}$ Physics Division, National Center for Theoretical Sciences, Hsinchu 300
}

(Received 26 December 2018; published 12 April 2019)

\begin{abstract}
We study the three-body antitriplet $\mathbf{B}_{\mathbf{c}} \rightarrow \mathbf{B}_{\mathbf{n}} M M^{\prime}$ decays with the $S U(3)$ flavor $\left[S U(3)_{f}\right]$ symmetry, where $\mathbf{B}_{\mathbf{c}}$ denotes the charmed baryon antitriplet of $\left(\Xi_{c}^{0},-\Xi_{c}^{+}, \Lambda_{c}^{+}\right)$, and $\mathbf{B}_{\mathbf{n}}$ and $M\left(M^{\prime}\right)$ represent baryon and meson octets, respectively. By considering only the $\mathrm{S}$-wave $M M^{\prime}$-pair contributions without resonance effects, the decays of $\mathbf{B}_{\mathbf{c}} \rightarrow \mathbf{B}_{\mathbf{n}} M M^{\prime}$ can be decomposed into irreducible forms with 11 parameters under $S U(3)_{f}$, which are fitted by the 14 existing data, resulting in a reasonable value of $\chi^{2} /$ d.o.f. $=2.8$ for the fit. Consequently, we find that the triangle sum rule of $\mathcal{A}\left(\Lambda_{c}^{+} \rightarrow n \bar{K}^{0} \pi^{+}\right)-\mathcal{A}\left(\Lambda_{c}^{+} \rightarrow p K^{-} \pi^{+}\right)-\sqrt{2} \mathcal{A}\left(\Lambda_{c}^{+} \rightarrow\right.$ $\left.p \bar{K}^{0} \pi^{0}\right)=0$ given by the isospin symmetry holds under $S U(3)_{f}$, where $\mathcal{A}$ stands for the decay amplitude. In addition, we predict that $\mathcal{B}\left(\Lambda_{c}^{+} \rightarrow n \pi^{+} \bar{K}^{0}\right)=(0.9 \pm 0.8) \times 10^{-2}$, which is 3-4 times smaller than the BESIII observation, indicating the existence of the resonant states. For the to-be-observed $\mathbf{B}_{\mathbf{c}} \rightarrow \mathbf{B}_{\mathbf{n}} M M^{\prime}$ decays, we compute the branching fractions with the $S U(3)_{f}$ amplitudes to be compared to the BESIII and $\mathrm{LHCb}$ measurements in the future.
\end{abstract}

DOI: 10.1103/PhysRevD.99.073003

\section{INTRODUCTION}

The three-body charmed baryon $\mathbf{B}_{\mathbf{c}} \rightarrow \mathbf{B}_{\mathbf{n}} M M^{\prime}$ decays have been recently searched by the experimental collaborations of Belle, BESIII, and LHCb, where $\mathbf{B}_{\mathbf{c}} \equiv$ $\left(\Xi_{c}^{0},-\Xi_{c}^{+}, \Lambda_{c}^{+}\right)$denotes the charmed baryon antitriplet and $\mathbf{B}_{\mathbf{n}}$ and $M^{(\prime)}$ correspond to the baryon and meson octets, respectively. For example, the decay of $\Lambda_{c}^{+} \rightarrow p K^{-} \pi^{+}$has been observed with high precision by Belle and BESIII [1,2], which improves the accuracy of the $\Lambda_{b}$ decays with $\Lambda_{c}^{+}$as one of the final states [3]. Also, the crucial information on the higher wave baryon resonances like $\Lambda(1405)$ has been extracted from the $\Sigma \pi$ invariant mass spectra of the $\Lambda_{c}^{+} \rightarrow$ $\Sigma \pi \pi$ decays [4]. The other area of interest comes from the test of the theoretical approach. For example, the first observation of $\Lambda_{c}^{+} \rightarrow n K_{s}^{0} \pi^{+}$has been used to examine the isospin relation [5], that is, $R(\Delta) \equiv \mathcal{A}\left(\Lambda_{c}^{+} \rightarrow n \bar{K}^{0} \pi^{+}\right)+$ $\mathcal{A}\left(\Lambda_{c}^{+} \rightarrow p K^{-} \pi^{+}\right)+\sqrt{2} \mathcal{A}\left(\Lambda_{c}^{+} \rightarrow p \bar{K}^{0} \pi^{0}\right)=0[6,7]{ }^{1}$

\footnotetext{
${ }^{1}$ To calculate the decay amplitude of $\mathcal{A}$, we use the conventions of $\left|\pi^{+}\right\rangle=-|11\rangle$ and $\left|\bar{K}^{0}\right\rangle=-\left|\frac{1}{2} \frac{1}{2}\right\rangle$, whereas $\left|\pi^{+}\right\rangle=|11\rangle$ and $\left|\bar{K}^{0}\right\rangle=\left|\frac{1}{2} \frac{1}{2}\right\rangle$ are taken in Refs. [6,7], resulting in the relation to be $R(\Delta) \equiv \mathcal{A}\left(\Lambda_{c}^{+} \rightarrow n \bar{K}^{0} \pi^{+}\right)-\mathcal{A}\left(\Lambda_{c}^{+} \rightarrow p K^{-} \pi^{+}\right)-\sqrt{2} \mathcal{A}\left(\Lambda_{c}^{+} \rightarrow\right.$ $\left.p \bar{K}^{0} \pi^{0}\right)=0$. However, the different signs in $R(\Delta)$ and other similar relations do not affect the physical consequences of these relations due to the arbitrariness of the phase of the amplitude.

Published by the American Physical Society under the terms of the Creative Commons Attribution 4.0 International license. Further distribution of this work must maintain attribution to the author(s) and the published article's title, journal citation, and DOI. Funded by SCOAP ${ }^{3}$.
}

Since the $\Lambda_{c}^{+} \rightarrow p K^{-} \pi^{+}$decay has diagrams similar to those of the doubly Cabibbo-suppressed $\Lambda_{c}^{+} \rightarrow p K^{+} \pi^{-}$one, we have the ratio of $\mathcal{B}\left(\Lambda_{c}^{+} \rightarrow p K^{+} \pi^{-}\right) / \mathcal{B}\left(\Lambda_{c}^{+} \rightarrow p K^{-} \pi^{+}\right)=$ $\mathcal{R}_{K \pi} \tan ^{4} \theta_{c}$ with $\mathcal{R}_{K \pi} \simeq 1.0$ and $\theta_{c}$, the Cabibbo angle, should hold. Nonetheless, the values of $\mathcal{R}_{K \pi}=0.82 \pm 0.12$ [8] and $0.58 \pm 0.06$ [9] have been measured by Belle and $\mathrm{LHCb}$, respectively, showing a possible deviation caused by an additional $W$-exchange amplitude for $\Lambda_{c}^{+} \rightarrow p K^{-} \pi^{+}$. As a result, the $\mathbf{B}_{\mathbf{c}} \rightarrow \mathbf{B}_{\mathbf{n}} M M^{\prime}$ decays are important for achieving a deeper insight into the hadronization of particle interactions.

In contrast with the abundant observations, there rarely exist systematic theoretical studies on the $\mathbf{B}_{\mathbf{c}} \rightarrow \mathbf{B}_{\mathbf{n}} M M^{\prime}$ decays, apart from those based on the isospin symmetry $[6,7]$. This is due to the fact that the scale of the charm quark mass $\left(m_{c}\right)$ is too large for the flavor $S U(3)\left[S U(3)_{f}\right]$ symmetry, but the theories based on the heavy quark expansion may not be valid as $m_{c}$ is not large enough. In addition, the factorization fails to work well in the charmed hadron decays $[6,10]$, whereas it is successfully used in the beauty hadron ones [11-13]. The alternative approaches for the charmed hadron decays have been shown in Refs. [14-19], which take into account the nonfactorizable effects. On the other hand, the $S U(3)_{f}$ symmetry has been tested as a useful tool both in the beauty and charmed hadron decays [20-27], particularly the twobody $\mathbf{B}_{\mathbf{c}} \rightarrow \mathbf{B}_{\mathbf{n}} M$ decays [6,28-37]. It is hence expected that the same symmetry can be applied to the three-body $\mathbf{B}_{\mathbf{c}} \rightarrow \mathbf{B}_{\mathbf{n}} M M^{\prime}$ decays. In this paper, we will relate the possible $\mathbf{B}_{\mathbf{c}} \rightarrow \mathbf{B}_{\mathbf{n}} M M^{\prime}$ decay processes with the $S U(3)_{f}$ 
parameters [28], by which the systematic numerical analysis can be performed for the first time. Under the $S U(3)_{f}$ symmetry, we will derive the relation of $\mathcal{R}(\Delta)=0$ and examine the value of $\mathcal{R}_{K \pi}$ from the ratio of $\mathcal{B}\left(\Lambda_{c}^{+} \rightarrow p K^{+} \pi^{-}\right) / \mathcal{B}\left(\Lambda_{c}^{+} \rightarrow p K^{-} \pi^{+}\right)$.

Our paper is organized as follows. We give the formalism in Sec. II, where the amplitudes for the three-body charmed baryon decays under the $S U(3)_{f}$ symmetry are presented. In Sec. III, we show our numerical results and discussions. Our conclusions are in Sec. IV.

\section{FORMALISM}

The three-body $\mathbf{B}_{\mathbf{c}} \rightarrow \mathbf{B}_{\mathbf{n}} M M^{\prime}$ decays can proceed through the charm quark decays of $c \rightarrow s u \bar{d}, \quad c \rightarrow$ $u d \bar{d}(u s \bar{s})$, and $c \rightarrow d u \bar{s}$, where $\mathbf{B}_{\mathbf{c}, \mathbf{n}}$ and $M^{\left({ }^{\prime}\right)}$ denote the baryon and meson states, respectively. Accordingly, the tree-level effective Hamiltonian is given by [38]

$\mathcal{H}_{\text {eff }}=\sum_{i=-,+} \frac{G_{F}}{\sqrt{2}} c_{i}\left[V_{c s} V_{u d} O_{i}+V_{c d} V_{u d} O_{i}^{\dagger}+V_{c d} V_{u s} O_{i}^{\prime}\right]$,

where $G_{F}$ is the Fermi constant, $c_{ \pm}$represent the Wilson coefficients, and $V_{i j}$ correspond to the CabibboKobayashi-Maskawa matrix elements, while $O_{\mp}, O_{\mp}^{\dagger}$, and $O_{\mp}^{\prime}$ are the four-quark operators, written as

$$
\begin{aligned}
O_{\mp}= & \frac{1}{2}[(\bar{u} d)(\bar{s} c) \mp(\bar{s} d)(\bar{u} c)], \\
O_{\mp}^{\dagger}= & \frac{1}{2}[(\bar{u} d)(\bar{d} c) \mp(\bar{d} d)(\bar{u} c)] \\
& -\frac{1}{2}[(\bar{u} s)(\bar{s} c) \mp(\bar{s} s)(\bar{u} c)], \\
O_{\mp}^{\prime}= & \frac{1}{2}[(\bar{u} s)(\bar{d} c) \mp(\bar{d} s)(\bar{u} c)],
\end{aligned}
$$

with $\left(\bar{q}_{1} q_{2}\right)\left(\bar{q}_{3} c\right) \equiv \bar{q}_{1} \gamma_{\mu}\left(1-\gamma_{5}\right) q_{2} \bar{q}_{3} \gamma^{\mu}\left(1-\gamma_{5}\right) c$. Here, the relation of $V_{c s} V_{u s}=-V_{c d} V_{u d}$ has been used for $O_{\mp}^{\dagger}$ to combine the $c \rightarrow u d \bar{d}$ and $c \rightarrow u s \bar{s}$ transitions. By means of the Cabibbo angle $\theta_{c}$, it is given that $\left(V_{c s} V_{u d}, V_{c d} V_{u d}, V_{c d} V_{u s}\right)=c_{c}^{2}\left(1,-t_{c},-t_{c}^{2}\right)$, where $\left(c_{c}, t_{c}\right) \equiv\left(\cos \theta_{c}, \tan \theta_{c}\right)$, such that the decays with $O_{\mp}$, $O_{\mp}^{\dagger}$, and $O_{\mp}^{\prime}$ are classified as the Cabibbo-favored (CF), Cabibbo-suppressed (CS), and doubly Cabibbo-suppressed (DCS) processes, respectively.

In Eq. (2), $\left(\bar{q}_{1} q_{2}\right)\left(\bar{q}_{3} c\right)$ can be rewritten as $\left(\bar{q}^{i} q_{k} \bar{q}^{j}\right) c$ with $q_{i}=(u, d, s)$ the triplet of 3 under the $S U(3)_{f}$ symmetry, by suppressing the Dirac and Lorentz indices. Furthermore, since $\left(\bar{q}^{i} q_{k} \bar{q}^{j}\right) c$ can be decomposed as the irreducible forms of $(\overline{3} \times 3 \times \overline{3}) c=\left(\overline{3}+\overline{3}^{\prime}+6+\overline{15}\right) c$, one derives that [28]

$$
\begin{aligned}
& O_{-(+)} \simeq \mathcal{O}_{6(\overline{15})}=\frac{1}{2}(\bar{u} d \bar{s} \mp \bar{s} d \bar{u}) c, \\
& O_{-(+)}^{\dagger} \simeq \mathcal{O}_{6(\overline{15})}^{\dagger}=\frac{1}{2}(\bar{u} d \bar{d} \mp \bar{d} d \bar{u}) c-\frac{1}{2}(\bar{u} s \bar{s} \mp \bar{s} s \bar{u}) c, \\
& O_{-(+)}^{\prime} \simeq \mathcal{O}_{6(\overline{15})}^{\prime}=\frac{1}{2}(\bar{u} s \bar{d} \mp \bar{d} s \bar{u}) c .
\end{aligned}
$$

Subsequently, the effective Hamiltonian in Eq. (1) has the expression under the $S U(3)_{f}$ symmetry, given by [31-34]

$$
\mathcal{H}_{\text {eff }}=\frac{G_{F}}{\sqrt{2}} c_{c}^{2}\left[c_{-} \frac{\epsilon^{i j l}}{2} H(6)_{l k}+c_{+} H(\overline{15})_{k}^{i j}\right] c,
$$

where $H(6, \overline{15})$ are presented as the tensor forms of $\left(\mathcal{O}_{6}^{(\dagger, \prime)}, \mathcal{O}_{\frac{15}{15}}^{(\dagger, \prime)}\right)$ in Eq. (3). Their nonzero entries are given by $[28,29]$

$$
\begin{aligned}
& H_{22}(6)=2, H_{2}^{13}(\overline{15})=H_{2}^{31}(\overline{15})=1, \\
& H_{23}(6)=H_{32}(6)=-2 t_{c}, H_{12}^{2}(\overline{15})=H_{21}^{2}(\overline{15})=t_{c}, \\
& H_{33}(6)=2 t_{c}^{2}, H_{3}^{12}(\overline{15})=H_{3}^{21}(\overline{15})=-t_{c}^{2}
\end{aligned}
$$

with $(i, j, k)$ for the quark indices. Correspondingly, the three lowest-lying charmed baryon states of $\mathbf{B}_{c}$ form an antitriplet of $\overline{3}$ to consist of $(d s-s d) c,(u s-s u) c$, and $(u d-d u) c$, and $\mathbf{B}_{n}(M)$ belongs to the baryon (meson) octet of 8 , which are written as

$$
\begin{aligned}
& \mathbf{B}_{c}=\left(\Xi_{c}^{0},-\Xi_{c}^{+}, \Lambda_{c}^{+}\right), \\
& \mathbf{B}_{n}=\left(\begin{array}{ccc}
\frac{1}{\sqrt{6}} \Lambda^{0}+\frac{1}{\sqrt{2}} \Sigma^{0} & \Sigma^{-} & \Xi^{-} \\
\Sigma^{+} & \frac{1}{\sqrt{6}} \Lambda^{0}-\frac{1}{\sqrt{2}} \Sigma^{0} & \Xi^{0} \\
p & n & -\sqrt{\frac{2}{3}} \Lambda^{0}
\end{array}\right), \\
& M=\left(\begin{array}{ccc}
\frac{1}{\sqrt{2}} \pi^{0}+\frac{1}{\sqrt{6}} \eta & \pi^{-} & K^{-} \\
\pi^{+} & -\frac{1}{\sqrt{2}} \pi^{0}+\frac{1}{\sqrt{6}} \eta & \bar{K}^{0} \\
K^{+} & K^{0} & -\sqrt{\frac{2}{3}} \eta
\end{array}\right),
\end{aligned}
$$

respectively.

Now, one is able to connect the octets of $\left(\mathbf{B}_{n}, M\right)_{j}^{i}$ and antitriplet of $\left(\mathbf{B}_{c}\right)_{i}$ to $\left(\epsilon^{i j l} H(6)_{l k}, H(\overline{15})_{k}^{i j}\right)$ in $\mathcal{H}_{\mathrm{eff}}$ of Eq. (4) to get the $S U(3)_{f}$ amplitudes. Since the Wilson coefficients are scale dependent, in the naive dimensional regularization scheme it is calculated that $\left(c_{-}, c_{+}\right)=$ $(1.78,0.76)$ at the scale $\mu=1 \mathrm{GeV}[39,40]$. The value of $\left(c_{-} / c_{+}\right)^{2} \simeq 5.5$ implies the suppressed branching ratios associated with $H(\overline{15})$. Hence, we follow Refs. $[6,31,36]$ to ignore the amplitudes from $H(\overline{15})$. By means of $\mathcal{A}\left(\mathbf{B}_{c} \rightarrow \mathbf{B}_{n} M M^{\prime}\right) \equiv\left(G_{F} / \sqrt{2}\right) T\left(\mathbf{B}_{c} \rightarrow \mathbf{B}_{n} M M^{\prime}\right)$, the T-amplitude of $\mathbf{B}_{c} \rightarrow \mathbf{B}_{n} M M^{\prime}$ can be derived as [28] 


$$
\begin{aligned}
T\left(\mathbf{B}_{c} \rightarrow \mathbf{B}_{n} M M\right)= & a_{1}\left(\overline{\mathbf{B}}_{n}\right)_{i}^{k}(M)_{l}^{m}\left(M^{\prime}\right)_{m}^{l} H(6)_{j k} T^{i j} \\
& +a_{2}\left(\overline{\mathbf{B}}_{n}\right)_{i}^{k}(M)_{j}^{m}\left(M^{\prime}\right)_{m}^{l} H(6)_{k l} T^{i j} \\
& +a_{3}\left(\overline{\mathbf{B}}_{n}\right)_{i}^{k}(M)_{k}^{m}\left(M^{\prime}\right)_{m}^{l} H(6)_{j l} T^{i j} \\
& +a_{4}\left(\overline{\mathbf{B}}_{n}\right)_{i}^{k}(M)_{j}^{l}\left(M^{\prime}\right)_{k}^{m} H(6)_{l m} T^{i j} \\
& +a_{5}\left(\overline{\mathbf{B}}_{n}\right)_{k}^{l}(M)_{j}^{m}\left(M^{\prime}\right)_{m}^{k} H(6)_{i l} T^{i j} \\
& +a_{6}\left(\overline{\mathbf{B}}_{n}\right)_{k}^{l}(M)_{j}^{m}\left(M^{\prime}\right)_{l}^{k} H(6)_{i m} T^{i j},
\end{aligned}
$$

with $T^{i j}=\left(\mathbf{B}_{\mathbf{c}}\right)_{a} \epsilon^{a i j}$, where $c_{c}^{2}$ and $c_{-}$in $\mathcal{H}_{\text {eff }}$ have been absorbed into the $S U(3)_{f}$ parameters $a_{i}(i=1,2, \ldots, 6)$. While there exists the relative orbital angular momentum $L$ between the two-meson states, we have assumed the S-wave $M M^{\prime}$-pair $(L=0)$ in the dominant amplitudes in Eq. (7), whereas the P-wave one $(L=1)$ is neglected. However, there are some cases in which the $S$-wave contributions vanish, but $\mathrm{P}$-wave ones are dominant, resulting in the other set of amplitudes to be studied elsewhere. For example, the decay of $\Lambda_{c}^{+} \rightarrow \Lambda \pi^{+} \pi^{0}$ with the measured branching ratio around $7.1 \%$ is mainly from the P-wave contribution.

The integration over the phase space of the three-body decay relies on the equation of [3]

$$
\Gamma=\int_{m_{12}^{2}} \int_{m_{23}^{2}} \frac{1}{(2 \pi)^{3}} \frac{\left|\mathcal{A}\left(\mathbf{B}_{c} \rightarrow \mathbf{B}_{n} M M^{\prime}\right)\right|^{2}}{32 m_{\mathbf{B}_{\mathbf{c}}}^{3}} d m_{12}^{2} d m_{23}^{2},
$$

where $m_{12}=p_{M}+p_{M^{\prime}}, m_{23}=p_{M^{\prime}}+p_{\mathbf{B}_{\mathrm{n}}}$, and $\mathcal{A}\left(\mathbf{B}_{c} \rightarrow\right.$ $\left.\mathbf{B}_{n} M M^{\prime}\right)$ is related to $T\left(\mathbf{B}_{c} \rightarrow \mathbf{B}_{n} M M^{\prime}\right)$ given in Eq. (7). In Tables I-III, we show the full expansions of $T\left(\Lambda_{c}^{+} \rightarrow\right.$ $\left.\mathbf{B}_{n} M M^{\prime}\right), \quad T\left(\Xi_{c}^{+} \rightarrow \mathbf{B}_{n} M M^{\prime}\right), \quad$ and $\quad T\left(\Xi_{c}^{0} \rightarrow \mathbf{B}_{n} M M^{\prime}\right)$, respectively. In general, the $S U(3)_{f}$ parameters depend on $m_{12}$ and $m_{23}$. However, all structures in the Dalitz plots come from the dynamical effects, such as those from the resonant states. Clearly, the squared amplitude in the Dalitz plot is almost structureless for the decay without the resonance. As a result, we treat our decay amplitudes as constants without energy dependences so that they can be factored out from the integrals as an approximation.

\section{NUMERICAL RESULTS AND DISCUSSIONS}

In the numerical analysis, we perform the minimum $\chi^{2}$ fit to examine if the $S U(3)_{f}$ symmetry is valid in the $\mathbf{B}_{\mathbf{c}} \rightarrow$ $\mathbf{B}_{n} M M^{\prime}$ decays. The equation of the $\chi^{2}$ fit is given by

$$
\chi^{2}=\sum_{i}\left(\frac{\mathcal{B}_{\mathrm{th}}^{i}-\mathcal{B}_{\mathrm{ex}}^{i}}{\sigma_{\mathrm{ex}}^{i}}\right)^{2},
$$

\begin{tabular}{|c|c|}
\hline CF mode & T-amp \\
\hline$\Sigma^{+} \pi^{0} \pi^{0}$ & $4 a_{1}+2 a_{2}+2 a_{3}+2 a_{4}-2 a_{5}$ \\
\hline$\Sigma^{+} \pi^{+} \pi^{-}$ & $4 a_{1}+2 a_{2}+2 a_{3}-2 a_{5}-2 a_{6}$ \\
\hline$\Sigma^{+} K^{0} \bar{K}^{0}$ & $4 a_{1}+2 a_{2}+2 a_{3}$ \\
\hline$\Sigma^{+} K^{+} K^{-}$ & $4 a_{1}-2 a_{5}$ \\
\hline$\Sigma^{+} \eta^{0} \eta^{0}$ & $4 a_{1}+\frac{2 a_{2}}{3}+\frac{2 a_{3}}{3}+\frac{2 a_{4}}{3}-\frac{2 a_{5}}{3}$ \\
\hline$\Sigma^{0} \pi^{0} \pi^{+}$ & $-2 a_{4}-2 a_{6}$ \\
\hline$\Sigma^{0} K^{+} \bar{K}^{0}$ & $\sqrt{2} a_{2}+\sqrt{2} a_{3}+\sqrt{2} a_{5}$ \\
\hline$\Sigma^{-} \pi^{+} \pi^{+}$ & $-4 a_{4}-4 a_{6}$ \\
\hline$\Xi^{0} \pi^{0} K^{+}$ & $-\sqrt{2} a_{5}$ \\
\hline$\Xi^{0} \pi^{+} K^{0}$ & $-2 a_{5}-2 a_{6}$ \\
\hline$\Xi^{-} \pi^{+} K^{+}$ & $-2 a_{6}$ \\
\hline$p \pi^{0} \bar{K}^{0}$ & $-\sqrt{2} a_{3}-\sqrt{2} a_{4}$ \\
\hline$p \pi^{+} K^{-}$ & $2 a_{3}-2 a_{6}$ \\
\hline$p \bar{K}^{0} \eta^{0}$ & $-\frac{\sqrt{6} a_{3}}{3}+\frac{\sqrt{6} a_{4}}{3}$ \\
\hline$n \pi^{+} \bar{K}^{0}$ & $-2 a_{4}-2 a_{6}$ \\
\hline$\Lambda^{0} \pi^{+} \eta^{0}$ & $-\frac{2 a_{2}}{3}+\frac{2 a_{3}}{3}-\frac{2 a_{5}}{3}-2 a_{6}$ \\
\hline$\Lambda^{0} K^{+} \bar{K}^{0}$ & $-\frac{\sqrt{6} a_{2}}{3}+\frac{\sqrt{6} a_{3}}{3}-\frac{\sqrt{6} a_{5}}{3}$ \\
\hline $\mathrm{CS}$ mode & $\mathrm{T}$-amp/t $t_{c}$ \\
\hline$\Sigma^{+} \pi^{0} K^{0}$ & $\sqrt{2} a_{2}+\sqrt{2} a_{3}+2 \sqrt{2} a_{4}$ \\
\hline$\Sigma^{+} \pi^{-} K^{+}$ & $-2 a_{2}-2 a_{3}+2 a_{6}$ \\
\hline$\Sigma^{+} K^{0} \eta^{0}$ & $\frac{\sqrt{6} a_{2}}{3}+\frac{\sqrt{6} a_{3}}{3}-\frac{2 \sqrt{6} a_{4}}{3}$ \\
\hline$\Sigma^{0} \pi^{+} K^{0}$ & $-\sqrt{2} a_{2}-\sqrt{2} a_{3}-2 \sqrt{2} a_{4}$ \\
\hline$\Sigma^{0} K^{+} \eta^{0}$ & $\frac{\sqrt{3} a_{2}}{3}+\frac{\sqrt{3} a_{3}}{3}-\frac{2 \sqrt{3} a_{4}}{3}$ \\
\hline$\Sigma^{-} \pi^{+} K^{+}$ & $4 a_{4}+2 a_{6}$ \\
\hline$p \pi^{0} \pi^{0}$ & $-4 a_{1}-2 a_{2}+2 a_{5}$ \\
\hline$p \pi^{0} \eta^{0}$ & $\frac{2 \sqrt{3} a_{2}}{3}-\frac{2 \sqrt{3} a_{4}}{3}+\frac{2 \sqrt{3} a_{5}}{3}$ \\
\hline$p \pi^{+} \pi^{-}$ & $-4 a_{1}-2 a_{2}+2 a_{5}$ \\
\hline$p K^{+} K^{-}$ & $-4 a_{1}-2 a_{3}+2 a_{5}+2 a_{6}$ \\
\hline$p \eta^{0} \eta^{0}$ & $-4 a_{1}-\frac{2 a_{2}}{3}-\frac{8 a_{3}}{3}+\frac{4 a_{4}}{3}+\frac{2 a_{5}}{3}$ \\
\hline$n \pi^{+} \eta^{0}$ & $\frac{2 \sqrt{6} a_{2}}{3}-\frac{2 \sqrt{6} a_{4}}{3}+\frac{2 \sqrt{6} a_{5}}{3}$ \\
\hline$n K^{+} \bar{K}^{0}$ & $2 a_{2}+2 a_{4}+2 a_{5}+2 a_{6}$ \\
\hline$\Lambda^{0} \pi^{0} K^{+}$ & $\frac{\sqrt{3} a_{2}}{3}-\frac{\sqrt{3} a_{3}}{3}-\frac{2 \sqrt{3} a_{5}}{3}$ \\
\hline$\Lambda^{0} \pi^{+} K^{0}$ & $\frac{\sqrt{6} a_{2}}{3}-\frac{\sqrt{6} a_{3}}{3}-\frac{2 \sqrt{6} a_{5}}{3}$ \\
\hline$\Lambda^{0} K^{+} \eta^{0}$ & $-\frac{a_{2}}{3}+\frac{a_{3}}{3}+\frac{2 a_{5}}{3}+2 a_{6}$ \\
\hline DCS mode & $\mathrm{T}$-amp $/ t_{c}^{2}$ \\
\hline$\Sigma^{+} K^{0} K^{0}$ & $4 a_{4}$ \\
\hline$\Sigma^{0} K^{0} K^{+}$ & $2 \sqrt{2} a_{4}$ \\
\hline$\Sigma^{-} K^{+} K^{+}$ & $-4 a_{4}$ \\
\hline$p \pi^{0} K^{0}$ & $-\sqrt{2} a_{2}$ \\
\hline$p \pi^{-} K^{+}$ & $2 a_{2}$ \\
\hline$p K^{0} \eta^{0}$ & $-\frac{\sqrt{6} a_{2}}{3}-\frac{2 \sqrt{6} a_{4}}{3}$ \\
\hline$n \pi^{0} K^{+}$ & $-\sqrt{2} a_{2}$ \\
\hline$n \pi^{+} K^{0}$ & $-2 a_{2}$ \\
\hline$n K^{+} \eta^{0}$ & $\frac{\sqrt{6} a_{2}}{3}+\frac{2 \sqrt{6} a_{4}}{3}$ \\
\hline
\end{tabular}

where $\mathcal{B}_{\text {th }}$ as $\mathcal{B}\left(\mathbf{B}_{\mathbf{c}} \rightarrow \mathbf{B}_{n} M M^{\prime}\right)$ is calculated by the $S U(3)_{f}$ parameters, and $\mathcal{B}_{\text {ex }}$ the experimental value in Table IV, with $\sigma$ the experimental error. With $\sin \theta_{c}=0.2248$ [3], one obtains that $t_{c}=0.2307$ as the input in Eq. (5). The $S U(3)_{f}$ parameters are written as
TABLE I. T-amplitudes of $\Lambda_{c}^{+} \rightarrow \mathbf{B}_{\mathbf{n}} M M^{\prime}$. 
TABLE II. T-amplitudes of $\Xi_{c}^{+} \rightarrow \mathbf{B}_{\mathbf{n}} M M^{\prime}$.

\begin{tabular}{lc}
\hline \hline CF mode & T-amp \\
\hline$\Sigma^{+} \pi^{0} \bar{K}^{0}$ & $-\sqrt{2} a_{2}-\sqrt{2} a_{4}$ \\
$\Sigma^{+} \pi^{+} K^{-}$ & $2 a_{2}$ \\
$\Sigma^{+} \bar{K}^{0} \eta^{0}$ & $-\frac{\sqrt{6} a_{2}}{3}+\frac{\sqrt{6} a_{4}}{3}$ \\
$\Sigma^{0} \pi^{+} \bar{K}^{0}$ & $\sqrt{2} a_{4}$ \\
$\Xi^{0} \pi^{0} \pi^{+}$ & $\sqrt{2} a_{4}$ \\
$\Xi^{0} \pi^{+} \eta^{0}$ & $-\frac{2 \sqrt{6} a_{2}}{3}-\frac{\sqrt{6} a_{4}}{3}$ \\
$\Xi^{0} K^{+} \bar{K}^{0}$ & $-2 a_{2}$ \\
$\Xi^{-} \pi^{+} \pi^{+}$ & $-4 a_{4}$ \\
$p \bar{K}^{0} \bar{K}^{0}$ & $4 a_{4}$ \\
$\Lambda^{0} \pi^{+} \bar{K}^{0}$ & $\sqrt{6} a_{4}$ \\
\hline
\end{tabular}

\begin{tabular}{|c|c|}
\hline CS mode & T-amp $/ t_{c}$ \\
\hline $\begin{array}{l}\Sigma^{+} \pi^{0} \pi^{0} \\
\Sigma^{+} \pi^{0} \eta^{0} \\
\Sigma^{+} \pi^{+} \pi^{-} \\
\Sigma^{+} K^{+} K^{-} \\
\Sigma^{+} \eta^{0} \eta^{0} \\
\Sigma^{0} \pi^{0} \pi^{+} \\
\Sigma^{0} \pi^{+} \eta^{0} \\
\Sigma^{0} K^{+} \bar{K}^{0} \\
\Sigma^{-} \pi^{+} \pi^{+} \\
\Xi^{0} \pi^{0} K^{+} \\
\Xi^{0} \pi^{+} K^{0} \\
\Xi^{0} K^{+} \eta^{0} \\
\Xi^{-} \pi^{+} K^{+} \\
p \pi^{0} \bar{K}^{0} \\
p \pi^{+} K^{-} \\
p \bar{K}^{0} \eta^{0} \\
n \pi^{+} \bar{K}^{0} \\
\Lambda^{0} \pi^{+} \eta^{0} \\
\Lambda^{0} K^{+} \bar{K}^{0}\end{array}$ & $\begin{array}{c}-4 a_{1}-2 a_{3}+2 a_{5} \\
\frac{2 \sqrt{3} a_{3}}{3}-\frac{2 \sqrt{3} a_{4}}{3}+\frac{2 \sqrt{3} a_{5}}{3} \\
-4 a_{1}-2 a_{3}+2 a_{5}+2 a_{6} \\
-4 a_{1}-2 a_{2}+2 a_{5} \\
-4 a_{1}-\frac{8 a_{2}}{3}-\frac{2 a_{3}}{3}+\frac{4 a_{4}}{3}+\frac{2 a_{5}}{3} \\
2 a_{6} \\
-\frac{2 \sqrt{3} a_{3}}{3}+\frac{2 \sqrt{3} a_{4}}{3}-\frac{2 \sqrt{3} a_{5}}{3} \\
-\sqrt{2} a_{3}-\sqrt{2} a_{4}-\sqrt{2} a_{5} \\
4 a_{6} \\
\sqrt{2} a_{2}-\sqrt{2} a_{4}+\sqrt{2} a_{5} \\
2 a_{2}+2 a_{4}+2 a_{5}+2 a_{6} \\
-\frac{\sqrt{6} a_{2}}{3}+\frac{\sqrt{6} a_{4}}{3}-\frac{\sqrt{6} a_{5}}{3} \\
4 a_{4}+2 a_{6} \\
\sqrt{2} a_{2}+\sqrt{2} a_{3} \\
-2 a_{2}-2 a_{3}+2 a_{6} \\
\frac{\sqrt{6} a_{2}}{3}+\frac{\sqrt{6} a_{3}}{3}+\frac{4 \sqrt{6} a_{4}}{3} \\
2 a_{6} \\
-\frac{4 a_{2}}{3}-\frac{2 a_{3}}{3}+2 a_{4}+\frac{2 a_{5}}{3}+2 a_{6} \\
-\frac{2 \sqrt{6} a_{2}}{3}-\frac{\sqrt{6} a_{3}}{3}-\sqrt{6} a_{4}+\frac{\sqrt{6} a_{5}}{3}\end{array}$ \\
\hline DCS mode & $\mathrm{T}$-amp/ $t_{c}^{2}$ \\
\hline $\begin{array}{l}\Sigma^{+} \pi^{0} K^{0} \\
\Sigma^{+} \pi^{-} K^{+} \\
\Sigma^{+} K^{0} \eta^{0} \\
\Sigma^{0} \pi^{0} K^{+} \\
\Sigma^{0} \pi^{+} K^{0} \\
\Sigma^{0} K^{+} \eta^{0} \\
\Sigma^{-} \pi^{+} K^{+} \\
\Xi^{0} K^{0} K^{+} \\
\Xi^{-} K^{+} K^{+} \\
p \pi^{0} \pi^{0} \\
p \pi^{0} \eta^{0} \\
p \pi^{+} \pi^{-} \\
p K^{0} \bar{K}^{0} \\
p K^{+} K^{-} \\
p \eta^{0} \eta^{0}\end{array}$ & $\begin{array}{c}-\sqrt{2} a_{3} \\
2 a_{3}-2 a_{6} \\
-\frac{\sqrt{6} a_{3}}{3}-\frac{2 \sqrt{6} a_{4}}{3} \\
a_{3}-2 a_{6} \\
\sqrt{2} a_{3} \\
-\frac{\sqrt{3} a_{3}}{3}-\frac{2 \sqrt{3} a_{4}}{3} \\
-2 a_{6} \\
-2 a_{4}-2 a_{6} \\
-4 a_{4}-4 a_{6} \\
4 a_{1}-2 a_{5} \\
-\frac{2 \sqrt{3} a_{5}}{3} \\
4 a_{1}-2 a_{5} \\
4 a_{1}+2 a_{2}+2 a_{3} \\
4 a_{1}+2 a_{2}+2 a_{3}-2 a_{5}-2 a_{6} \\
4 a_{1}+\frac{8 a_{2}}{3}+\frac{8 a_{3}}{3}+\frac{8 a_{4}}{3}-\frac{2 a_{5}}{3}\end{array}$ \\
\hline
\end{tabular}

(Table continued)
TABLE II. (Continued)

\begin{tabular}{lc}
\hline \hline DCS mode & T-amp/t $t_{c}^{2}$ \\
\hline$n \pi^{+} \eta^{0}$ & $-\frac{2 \sqrt{6} a_{5}}{3}$ \\
$n K^{+} \bar{K}^{0}$ & $-2 a_{5}-2 a_{6}$ \\
$\Lambda^{0} \pi^{0} K^{+}$ & $\frac{2 \sqrt{3} a_{2}}{3}+\frac{\sqrt{3} a_{3}}{3}+\frac{2 \sqrt{3} a_{5}}{3}$ \\
$\Lambda^{0} \pi^{+} K^{0}$ & $\frac{2 \sqrt{6} a_{2}}{3}+\frac{\sqrt{6} a_{3}}{3}+\frac{2 \sqrt{6} a_{5}}{3}$ \\
\hline \hline
\end{tabular}

TABLE III. T-amplitudes of $\Xi_{c}^{0} \rightarrow \mathbf{B}_{\mathbf{n}} M M^{\prime}$.

\begin{tabular}{|c|c|}
\hline CF mode & T-amp \\
\hline$\Sigma^{+} \pi^{0} K^{-}$ & $\sqrt{2} a_{5}$ \\
\hline$\Sigma^{+} \pi^{-} \bar{K}^{0}$ & $2 a_{5}+2 a_{6}$ \\
\hline$\Sigma^{+} K^{-} \eta^{0}$ & $-\frac{\sqrt{6} a_{5}}{3}$ \\
\hline$\Sigma^{0} \pi^{0} \bar{K}^{0}$ & $a_{2}+a_{4}+a_{5}+2 a_{6}$ \\
\hline$\Sigma^{0} \pi^{+} K^{-}$ & $-\sqrt{2} a_{2}-\sqrt{2} a_{5}$ \\
\hline$\Sigma^{0} \bar{K}^{0} \eta^{0}$ & $\frac{\sqrt{3} a_{2}}{3}-\frac{\sqrt{3} a_{4}}{3}+\frac{\sqrt{3} a_{5}}{3}$ \\
\hline$\Sigma^{-} \pi^{+} \bar{K}^{0}$ & $2 a_{4}+2 a_{6}$ \\
\hline$\Xi^{0} \pi^{0} \eta^{0}$ & $\frac{2 \sqrt{3} a_{2}}{3}+\frac{2 \sqrt{3} a_{3}}{3}+\frac{2 \sqrt{3} a_{4}}{3}$ \\
\hline$\Xi^{0} \pi^{+} \pi^{-}$ & $-4 a_{1}-2 a_{2}-2 a_{3}$ \\
\hline$\Xi^{0} K^{0} \bar{K}^{0}$ & $-2\left(2 a_{1}+a_{2}+a_{3}-a_{5}-a_{6}\right)$ \\
\hline$\Xi^{0} K^{+} K^{-}$ & $-4 a_{1}+2 a_{5}$ \\
\hline$\Xi^{0} \eta^{0} \eta^{0}$ & $-2\left(2 a_{1}+\frac{a_{2}}{3}+\frac{a_{3}}{3}+\frac{a_{4}}{3}-\frac{4 a_{5}}{3}\right)$ \\
\hline$\Xi^{-} \pi^{0} \pi^{+}$ & $\sqrt{2} a_{4}$ \\
\hline$\Xi^{-} \pi^{+} \eta^{0}$ & $-\frac{2 \sqrt{6} a_{3}}{3}-\frac{\sqrt{6} a_{4}}{3}$ \\
\hline$\Xi^{-} K^{+} \bar{K}^{0}$ & $-2 a_{3}+2 a_{6}$ \\
\hline$p K^{-} \bar{K}^{0}$ & $2 a_{6}$ \\
\hline$n \bar{K}^{0} \bar{K}^{0}$ & $4 a_{4}+4 a_{6}$ \\
\hline$\Lambda^{0} \pi^{0} \bar{K}^{0}$ & $-\sqrt{3}\left(\frac{a_{2}}{3}+\frac{2 a_{3}}{3}+a_{4}+\frac{a_{5}}{3}\right)$ \\
\hline$\Lambda^{0} \pi^{+} K^{-}$ & $\frac{\sqrt{6} a_{2}}{3}+\frac{2 \sqrt{6} a_{3}}{3}+\frac{\sqrt{6} a_{5}}{3}$ \\
\hline CS mode & $\mathrm{T}$-amp/ $t_{c}$ \\
\hline$\Sigma^{+} \pi^{0} \pi^{-}$ & $-\sqrt{2} a_{6}$ \\
\hline$\Sigma^{+} \pi^{-} \eta^{0}$ & $\frac{2 \sqrt{6} a_{5}}{3}+\sqrt{6} a_{6}$ \\
\hline$\Sigma^{+} K^{0} K^{-}$ & $2 a_{5}$ \\
\hline$\Sigma^{0} \pi^{0} \pi^{0}$ & $2 \sqrt{2} a_{1}+\sqrt{2} a_{3}-\sqrt{2} a_{5}-2 \sqrt{2} a_{6}$ \\
\hline$\Sigma^{0} \pi^{0} \eta^{0}$ & $-\frac{\sqrt{6} a_{3}}{3}+\frac{\sqrt{6} a_{4}}{3}+\frac{\sqrt{6} a_{5}}{3}+\sqrt{6} a_{6}$ \\
\hline$\Sigma^{0} \pi^{+} \pi^{-}$ & $2 \sqrt{2} a_{1}+\sqrt{2} a_{3}-\sqrt{2} a_{5}$ \\
\hline$\Sigma^{0} K^{0} \bar{K}^{0}$ & $\sqrt{2}\left(2 a_{1}+a_{2}+a_{3}+a_{4}-a_{5}\right)$ \\
\hline$\Sigma^{0} K^{+} K^{-}$ & $2 \sqrt{2} a_{1}+\sqrt{2} a_{2}$ \\
\hline$\Sigma^{0} \eta^{0} \eta^{0}$ & $\sqrt{2}\left(2 a_{1}+\frac{4 a_{2}}{3}+\frac{a_{3}}{3}-\frac{2 a_{4}}{3}-\frac{a_{5}}{3}\right)$ \\
\hline$\Sigma^{-} \pi^{0} \pi^{+}$ & $-\sqrt{2} a_{6}$ \\
\hline$\Sigma^{-} \pi^{+} \eta^{0}$ & $-\frac{2 \sqrt{6} a_{3}}{3}+\frac{2 \sqrt{6} a_{4}}{3}+\sqrt{6} a_{6}$ \\
\hline$\Sigma^{-} K^{+} \bar{K}^{0}$ & $-2 a_{3}-2 a_{4}$ \\
\hline$\Xi^{0} \pi^{-} K^{+}$ & $2 a_{2}+2 a_{3}+2 a_{5}$ \\
\hline$\Xi^{0} K^{0} \eta^{0}$ & $\sqrt{6}\left(-\frac{a_{2}}{3}-\frac{a_{3}}{3}+\frac{2 a_{4}}{3}-\frac{a_{5}}{3}+a_{6}\right)$ \\
\hline$\Xi^{-} \pi^{0} K^{+}$ & $\sqrt{2} a_{3}-\sqrt{2} a_{4}-\sqrt{2} a_{6}$ \\
\hline
\end{tabular}

(Table continued) 
TABLE III. (Continued)

\begin{tabular}{|c|c|}
\hline CS mode & $\mathrm{T}$-amp/t $t_{c}$ \\
\hline$\Xi^{-} \pi^{+} K^{0}$ & $2 a_{3}+2 a_{4}$ \\
\hline$p \pi^{0} K^{-}$ & $-\sqrt{2} a_{5}-\sqrt{2} a_{6}$ \\
\hline$p \pi^{-} \bar{K}^{0}$ & $-2 a_{5}$ \\
\hline$p K^{-} \eta^{0}$ & $\frac{\sqrt{6} a_{5}}{3}+\sqrt{6} a_{6}$ \\
\hline$n \pi^{0} \bar{K}^{0}$ & $\sqrt{2} a_{2}+\sqrt{2} a_{3}+\sqrt{2} a_{5}-\sqrt{2} a_{6}$ \\
\hline$n \pi^{+} K^{-}$ & $-2 a_{2}-2 a_{3}-2 a_{5}$ \\
\hline$n \bar{K}^{0} \eta^{0}$ & $\sqrt{6}\left(\frac{a_{2}}{3}+\frac{a_{3}}{3}+\frac{4 a_{4}}{3}+\frac{a_{5}}{3}+a_{6}\right)$ \\
\hline$\Lambda^{0} \pi^{0} \pi^{0}$ & $\sqrt{6}\left(-2 a_{1}-\frac{2 a_{2}}{3}-\frac{a_{3}}{3}+\frac{a_{5}}{3}\right)$ \\
\hline$\Lambda^{0} \pi^{0} \eta^{0}$ & $\sqrt{2}\left(\frac{2 a_{2}}{3}+\frac{a_{3}}{3}-a_{4}-\frac{a_{5}}{3}-a_{6}\right)$ \\
\hline$\Lambda^{0} \pi^{+} \pi^{-}$ & $\sqrt{6}\left(-2 a_{1}-\frac{2 a_{2}}{3}-\frac{a_{3}}{3}+\frac{a_{5}}{3}\right)$ \\
\hline$\Lambda^{0} K^{0} \bar{K}^{0}$ & $\sqrt{6}\left(-2 a_{1}-a_{2}-a_{3}-a_{4}+a_{5}\right)$ \\
\hline$\Lambda^{0} K^{+} K^{-}$ & $\sqrt{6}\left(-2 a_{1}-\frac{a_{2}}{3}-\frac{2 a_{3}}{3}+\frac{2 a_{5}}{3}\right)$ \\
\hline$\Lambda^{0} \eta^{0} \eta^{0}$ & $\sqrt{6}\left(-2 a_{1}-\frac{2 a_{2}}{3}-a_{3}+\frac{2 a_{4}}{3}+a_{5}+2 a_{6}\right)$ \\
\hline DCS mode & $\mathrm{T}$-amp $/ t_{c}^{2}$ \\
\hline$\Sigma^{+} \pi^{-} K^{0}$ & $-2 a_{6}$ \\
\hline$\Sigma^{0} \pi^{0} K^{0}$ & $a_{3}-2 a_{6}$ \\
\hline$\Sigma^{0} \pi^{-} K^{+}$ & $-\sqrt{2} a_{3}$ \\
\hline$\Sigma^{0} K^{0} \eta^{0}$ & $\frac{\sqrt{3} a_{3}}{3}+\frac{2 \sqrt{3} a_{4}}{3}$ \\
\hline$\Sigma^{-} \pi^{0} K^{+}$ & $\sqrt{2} a_{3}$ \\
\hline$\Sigma^{-} \pi^{+} K^{0}$ & $2 a_{3}-2 a_{6}$ \\
\hline$\Sigma^{-} K^{+} \eta^{0}$ & $-\frac{\sqrt{6} a_{3}}{3}-\frac{2 \sqrt{6} a_{4}}{3}$ \\
\hline$\Xi^{0} K^{0} K^{0}$ & $-4 a_{4}-4 a_{6}$ \\
\hline$\Xi^{-} K^{0} K^{+}$ & $-2 a_{4}-2 a_{6}$ \\
\hline$p \pi^{-} \eta^{0}$ & $-\frac{2 \sqrt{6} a_{5}}{3}$ \\
\hline$p K^{0} K^{-}$ & $-2 a_{5}-2 a_{6}$ \\
\hline$n \pi^{0} \pi^{0}$ & $4 a_{1}-2 a_{5}$ \\
\hline$n \pi^{0} \eta^{0}$ & $\frac{2 \sqrt{3} a_{5}}{3}$ \\
\hline$n \pi^{+} \pi^{-}$ & $4 a_{1}-2 a_{5}$ \\
\hline$n K^{0} \bar{K}^{0}$ & $2\left(2 a_{1}+a_{2}+a_{3}-a_{5}-a_{6}\right)$ \\
\hline$n K^{+} K^{-}$ & $4 a_{1}+2 a_{2}+2 a_{3}$ \\
\hline$n \eta^{0} \eta^{0}$ & $4 a_{1}+\frac{8 a_{2}}{3}+\frac{8 a_{3}}{3}+\frac{8 a_{4}}{3}-\frac{2 a_{5}}{3}$ \\
\hline$\Lambda^{0} \pi^{0} K^{0}$ & $-\sqrt{3}\left(\frac{2 a_{2}}{3}+\frac{a_{3}}{3}+\frac{2 a_{5}}{3}\right)$ \\
\hline$\Lambda^{0} \pi^{-} K^{+}$ & $\sqrt{6}\left(\frac{2 a_{2}}{3}+\frac{a_{3}}{3}+\frac{2 a_{5}}{3}\right)$ \\
\hline
\end{tabular}

$$
a_{1}, a_{2} e^{i \delta_{a_{2}}}, a_{3} e^{i \delta_{a_{3}}}, a_{4} e^{i \delta_{a_{4}}}, a_{5} e^{i \delta_{a_{5}}}, a_{6} e^{i \delta_{a_{6}}},
$$

where the phases $\delta_{a_{2,3, \ldots 6}}$ are due to the nature of complex numbers associated with $a_{i}$, while $a_{1}$ can be relatively real. This leads to the reduced 11 parameters to be extracted with 14 data inputs in Table IV, where the fitting values of $a_{i}$ and $\delta_{a_{i}}$ are shown in Table V. We find that $\chi^{2} /$ d.o.f. $=8.4 / 3=2.8$ with d.o.f. representing the degree of freedom, and we reproduce the branching ratios in the third column of Table IV in order to compare them to the data. Note that in calculating the decay branching ratios, we have treated our $S U(3)_{f}$ parameters as independent ones,
TABLE IV. The data of $\mathcal{B}\left(\Lambda_{c}^{+} \rightarrow \mathbf{B}_{\mathbf{n}} M M\right)$ from the PDG [3], except for $\mathcal{B}\left(\Lambda_{c}^{+} \rightarrow \Sigma^{+} \pi^{0} \pi^{0}, p K^{+} \pi^{-}\right)[4,9]$.

\begin{tabular}{lcc}
\hline \hline Branching ratios & Data & Our results \\
\hline $10^{2} \mathcal{B}\left(\Lambda_{c}^{+} \rightarrow p K^{-} \pi^{+}\right)$ & $3.4 \pm 0.4$ & $3.3 \pm 1.0$ \\
$10^{2} \mathcal{B}\left(\Lambda_{c}^{+} \rightarrow p \bar{K}^{0} \eta\right)$ & $1.6 \pm 0.4$ & $0.9 \pm 0.1$ \\
$10^{3} \mathcal{B}\left(\Lambda_{c}^{+} \rightarrow \Lambda^{0} K^{+} \bar{K}^{0}\right)$ & $5.6 \pm 1.1$ & $5.7 \pm 1.1$ \\
$10^{2} \mathcal{B}\left(\Lambda_{c}^{+} \rightarrow \Lambda^{0} \pi^{+} \eta\right)$ & $2.2 \pm 0.5$ & $2.1 \pm 0.9$ \\
$10^{2} \mathcal{B}\left(\Lambda_{c}^{+} \rightarrow \Sigma^{+} \pi^{+} \pi^{-}\right)$ & $4.4 \pm 0.3$ & $4.4 \pm 3.5$ \\
$10^{2} \mathcal{B}\left(\Lambda_{c}^{+} \rightarrow \Sigma^{-} \pi^{+} \pi^{+}\right)$ & $1.9 \pm 0.2$ & $1.9 \pm 1.3$ \\
$10^{2} \mathcal{B}\left(\Lambda_{c}^{+} \rightarrow \Sigma^{0} \pi^{+} \pi^{0}\right)$ & $2.2 \pm 0.8$ & $1.0 \pm 0.8$ \\
$10^{2} \mathcal{B}\left(\Lambda_{c}^{+} \rightarrow \Sigma^{+} \pi^{0} \pi^{0}\right)$ & $1.3 \pm 0.1$ & $1.3 \pm 1.3$ \\
$10^{3} \mathcal{B}\left(\Lambda_{c}^{+} \rightarrow \Sigma^{+} K^{+} \pi^{-}\right)$ & $2.1 \pm 0.6$ & $3.0 \pm 0.4$ \\
$10^{3} \mathcal{B}\left(\Lambda_{c}^{+} \rightarrow \Xi^{-} K^{+} \pi^{+}\right)$ & $6.2 \pm 0.6$ & $6.3 \pm 0.6$ \\
$10^{2} \mathcal{B}\left(\Xi_{c}^{+} \rightarrow \Xi^{-} \pi^{+} \pi^{+}\right)$ & $6.1 \pm 3.1$ & $7.2 \pm 2.0$ \\
$10^{3} \mathcal{B}\left(\Lambda_{c}^{+} \rightarrow p \pi^{-} \pi^{+}\right)$ & $4.2 \pm 0.4$ & $4.7 \pm 1.6$ \\
$10^{4} \mathcal{B}\left(\Lambda_{c}^{+} \rightarrow p K^{-} K^{+}\right)$ & $5.2 \pm 1.2$ & $5.1 \pm 2.1$ \\
$10^{4} \mathcal{B}\left(\Lambda_{c}^{+} \rightarrow p K^{+} \pi^{-}\right)$ & $1.0 \pm 0.1$ & $1.0 \pm 0.1$ \\
\hline \hline
\end{tabular}

which may result in overestimated error ranges in our results.

To determine the $S U(3)_{f}$ parameters, we use the nonresonant parts of $\Lambda_{c}^{+} \rightarrow p K^{-} \pi^{+}$from the PDG [3]. Note that the resonant $\Lambda_{c}^{+} \rightarrow p\left(\bar{K}^{* 0} \rightarrow\right) K^{-} \pi^{+}$, $K^{-}\left(\Delta(1232)^{++} \rightarrow\right) p \pi^{+}$, and $\pi^{+}(\Lambda(1520) \rightarrow) p K^{-}$contributions are separated from its total branching ratio. In addition, the decay of $\Lambda_{c}^{+} \rightarrow p K^{-} K^{+}$is free from the resonant one of $\Lambda_{c}^{+} \rightarrow p(\phi \rightarrow) K^{-} K^{+}$. For the other $\Lambda_{c}^{+}$decays in Table IV, some of their resonant parts might be present, but taken to be small, such as $\mathcal{B}\left(\Lambda_{c}^{+} \rightarrow\right.$ $\left.\Sigma^{+}\left(\rho^{0} \rightarrow\right) \pi^{+} \pi^{-}\right)<1.7 \%$ [3], which should be insensitive to the fit. We hence use their total branching ratios, instead of excluding the resonant contributions. The $\Xi_{c}^{0,+} \rightarrow \mathbf{B}_{\mathbf{n}} M M^{\prime}$ decays are partially observed, such that we can barely use their data. Nonetheless, in terms of $T\left(\Lambda_{c}^{+} \rightarrow \Xi^{-} K^{+} \pi^{+}\right)=1 /\left(-2 t_{c}\right) T\left(\Xi_{c}^{+} \rightarrow \Sigma^{-} \pi^{+} \pi^{+}\right)=$ $-2 a_{6}$ and the data of $\mathcal{B}\left(\Lambda_{c}^{+} \rightarrow \Xi^{-} K^{+} \pi^{+}\right)$, we obtain $\mathcal{B}\left(\Xi_{c}^{+} \rightarrow \Sigma^{-} \pi^{+} \pi^{+}\right)=(1.1 \pm 0.1) \times 10^{-2}$, by which the observed ratio of $\mathcal{B}\left(\Xi_{c}^{+} \rightarrow \Sigma^{-} \pi^{+} \pi^{+}\right) / \mathcal{B}\left(\Xi_{c}^{+} \rightarrow \Xi^{-} \pi^{+} \pi^{+}\right)=$ $0.18 \pm 0.09$ and it leads to $\mathcal{B}\left(\Xi_{c}^{+} \rightarrow \Xi^{-} \pi^{+} \pi^{+}\right)=(6.1 \pm$ $3.1) \times 10^{-2}$ as given in Table IV.

TABLE V. Fitting results for $a_{i}$ and $\delta_{a_{i}}$.

\begin{tabular}{cccc}
\hline \hline$a_{i}$ & Result $\left(\mathrm{GeV}^{2}\right)$ & $\delta_{a_{i}}$ & Result \\
\hline$a_{1}$ & $9.1 \pm 0.6$ & $\ldots$ & $\ldots$ \\
$a_{2}$ & $4.6 \pm 0.2$ & $\delta_{a_{2}}$ & $164^{\circ} \pm 5^{\circ}$ \\
$a_{3}$ & $8.2 \pm 0.3$ & $\delta_{a_{3}}$ & $135^{\circ} \pm 5^{\circ}$ \\
$a_{4}$ & $2.9 \pm 0.4$ & $\delta_{a_{4}}$ & $-30^{\circ} \pm 13^{\circ}$ \\
$a_{5}$ & $15.4 \pm 1.4$ & $\delta_{a_{5}}$ & $24^{\circ} \pm 3^{\circ}$ \\
$a_{6}$ & $4.2 \pm 0.2$ & $\delta_{a_{6}}$ & $120^{\circ} \pm 10^{\circ}$ \\
\hline \hline
\end{tabular}


TABLE VI. Numerical results for the branching ratios of $\Lambda_{c}^{+} \rightarrow \mathbf{B}_{\mathbf{n}} M M^{\prime}$, where $\mathcal{B}_{\mathbf{B}_{\mathbf{n}} M M^{\prime}} \equiv \mathcal{B}\left(\Lambda_{c}^{+} \rightarrow \mathbf{B}_{\mathbf{n}} M M^{\prime}\right)$.

\begin{tabular}{|c|c|}
\hline CF mode & Our result \\
\hline $\begin{array}{l}10^{2} \mathcal{B}_{\Sigma^{+} \pi^{0} \eta^{0}} \\
10^{3} \mathcal{B}_{\Sigma^{+} K^{0} \bar{K}^{0}} \\
10^{3} \mathcal{B}_{\Sigma^{+} K^{+} K^{-}} \\
10^{7} \mathcal{B}_{\Sigma^{+} \eta^{0} \eta^{0}} \\
10^{2} \mathcal{B}_{\Sigma^{0} \pi^{+} \eta^{0}} \\
10^{2} \mathcal{B}_{\Sigma^{0} K^{+} \bar{K}^{0}} \\
10^{2} \mathcal{B}_{\Xi^{0} \pi^{0} K^{+}} \\
10^{2} \mathcal{B}_{\Xi^{0}} \pi^{+} K^{0} \\
10^{2} \mathcal{B}_{p \pi^{0} \bar{K}^{0}} \\
10^{2} \mathcal{B}_{n \pi^{+} \bar{K}^{0}}\end{array}$ & $\begin{array}{l}3.5 \pm 0.8 \\
5.2 \pm 1.2 \\
3.0 \pm 0.7 \\
2.8 \pm 0.6 \\
3.4 \pm 0.8 \\
0.5 \pm 0.1 \\
4.5 \pm 0.8 \\
8.7 \pm 1.7 \\
2.8 \pm 0.6 \\
0.9 \pm 0.8\end{array}$ \\
\hline CS mode & Our result \\
\hline $\begin{array}{l}10^{4} \mathcal{B}_{\Sigma^{+} \pi^{0} K^{0}} \\
10^{5} \mathcal{B}_{\Sigma^{+} K^{0} \eta^{0}} \\
10^{3} \mathcal{B}_{\Sigma^{0} \pi^{0} K^{+}} \\
10^{4} \mathcal{B}_{\Sigma^{0} \pi^{+} K^{0}} \\
10^{5} \mathcal{B}_{\Sigma^{0} K^{+} \eta^{0}} \\
10^{4} \mathcal{B}_{\Sigma^{-} \pi^{+} K^{+}} \\
10^{3} \mathcal{B}_{p \pi^{0} \pi^{0}} \\
10^{3} \mathcal{B}_{p \pi^{0} \eta^{0}} \\
10^{3} \mathcal{B}_{p k^{0} \bar{K}^{0}} \\
10^{4} \mathcal{B}_{p \eta^{0} \eta^{0}} \\
10^{3} \mathcal{B}_{n \pi^{+} \eta^{0}} \\
10^{3} \mathcal{B}_{n K^{+} \bar{K}^{0}} \\
10^{3} \mathcal{B}_{\Lambda^{0} \pi^{0} K^{+}} \\
10^{3} \mathcal{B}_{\Lambda^{0} \pi^{+} K^{0}} \\
10^{4} \mathcal{B}_{\Lambda^{0} K^{+} \eta^{0}}\end{array}$ & $\begin{array}{l}8.6 \pm 2.6 \\
3.5 \pm 0.4 \\
1.2 \pm 0.3 \\
8.3 \pm 2.5 \\
1.8 \pm 0.2 \\
3.3 \pm 2.3 \\
2.4 \pm 0.8 \\
3.7 \pm 0.9 \\
4.3 \pm 1.0 \\
4.7 \pm 1.0 \\
7.3 \pm 1.8 \\
5.9 \pm 1.3 \\
4.5 \pm 0.8 \\
8.8 \pm 1.5 \\
1.9 \pm 0.6\end{array}$ \\
\hline DCS mode & Our result \\
\hline $\begin{array}{l}10^{6} \mathcal{B}_{\Sigma^{+} K^{0} K^{0}} \\
10^{6} \mathcal{B}_{\Sigma^{0} K^{0} K^{+}} \\
10^{6} \mathcal{B}_{\Sigma^{-} K^{+} K^{+}} \\
10^{5} \mathcal{B}_{p \pi^{0} K^{0}} \\
10^{5} \mathcal{B}_{n \pi^{0} K^{+}} \\
10^{4} \mathcal{B}_{n \pi^{+} K^{0}}\end{array}$ & $\begin{array}{l}2.0 \pm 0.5 \\
2.0 \pm 0.6 \\
2.0 \pm 0.5 \\
5.0 \pm 0.5 \\
5.0 \pm 0.5 \\
1.0 \pm 0.1\end{array}$ \\
\hline
\end{tabular}

TABLE VII. Numerical results for the branching ratios of $\Xi_{c}^{+} \rightarrow \mathbf{B}_{\mathbf{n}} M M^{\prime}$, where $\mathcal{B}_{\mathbf{B}_{\mathbf{n}} M M^{\prime}} \equiv \mathcal{B}\left(\Xi_{c}^{+} \rightarrow \mathbf{B}_{\mathbf{n}} M M^{\prime}\right)$.

\begin{tabular}{ll}
\hline \hline CF mode & Our result \\
\hline $10^{3} \mathcal{B}_{\Sigma^{+} \pi^{0} \bar{K}^{0}}$ & $5.4 \pm 4.0$ \\
$10^{2} \mathcal{B}_{\Sigma^{+} \pi^{+} K^{-}}$ & $6.1 \pm 0.6$ \\
$10^{3} \mathcal{B}_{\Sigma^{+} \bar{K}^{0} \eta^{0}}$ & $4.6 \pm 0.6$ \\
$10^{2} \mathcal{B}_{\Sigma^{0} \pi^{+} \bar{K}^{0}}$ & $1.2 \pm 0.3$ \\
\hline
\end{tabular}

(Table continued)
TABLE VII. (Continued)

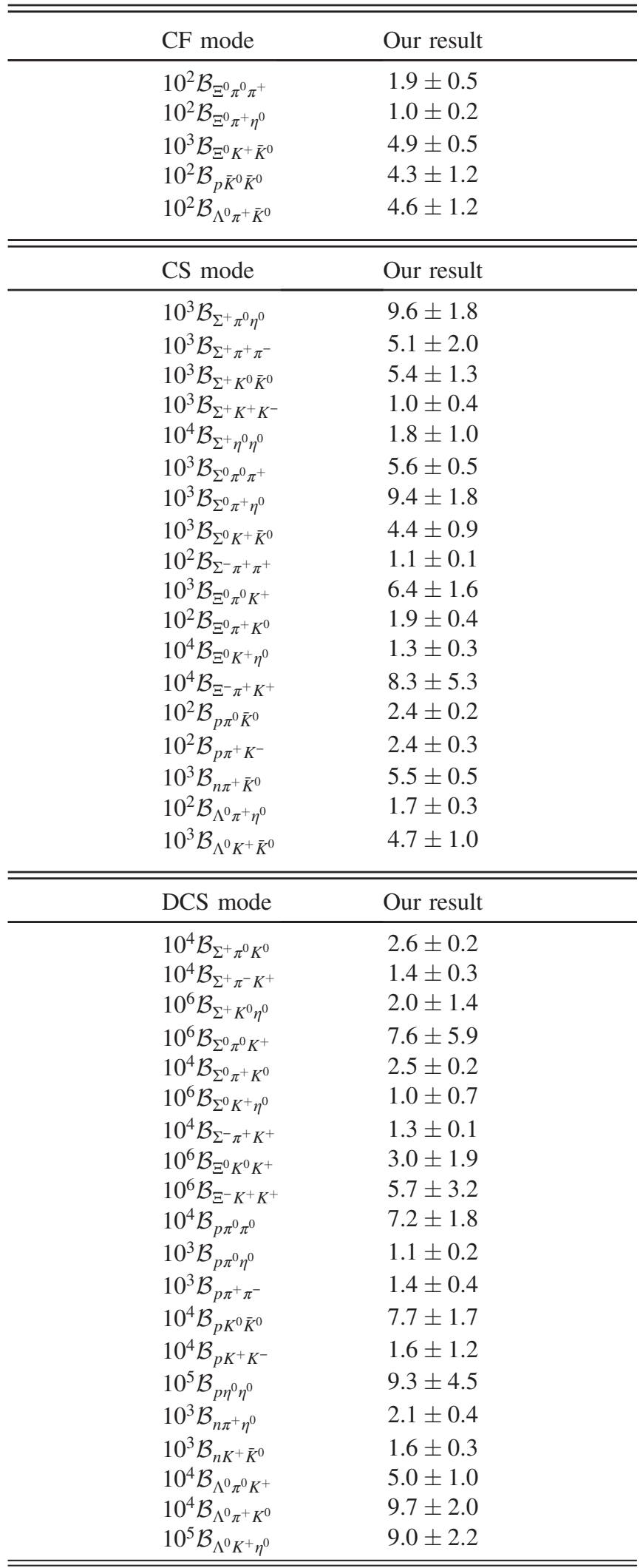


With $\chi^{2} /$ d.o.f. being 2.8 in Table $\mathrm{V}$, it turns out to be a reasonable fit, so that the $S U(3)_{f}$ symmetry with the reduced parameters can be used to explain the three-body $\mathbf{B}_{\mathbf{c}} \rightarrow \mathbf{B}_{\mathbf{n}} M M^{\prime}$ decays. The relations of $T\left(\Lambda_{c}^{+} \rightarrow n \bar{K}^{0} \pi^{+}\right)=$ $T\left(\Lambda_{c}^{+} \rightarrow \Sigma^{0} \pi^{0} \pi^{+}\right) \quad$ and $\quad T\left(\Lambda_{c}^{+} \rightarrow \Sigma^{0} \pi^{0} \pi^{+}\right)=T\left(\Lambda_{c}^{+} \rightarrow\right.$ $\left.\Sigma^{-} \pi^{+} \pi^{+}\right) / 2$ yield

$$
\begin{aligned}
\mathcal{B}\left(\Lambda_{c}^{+} \rightarrow n \bar{K}^{0} \pi^{+}\right) & \simeq \mathcal{B}\left(\Lambda_{c}^{+} \rightarrow \Sigma^{0} \pi^{0} \pi^{+}\right) \\
& \simeq \frac{1}{2} \mathcal{B}\left(\Lambda_{c}^{+} \rightarrow \Sigma^{-} \pi^{+} \pi^{+}\right),
\end{aligned}
$$

which agrees with our numerical analysis. Note that the calculation of $\mathcal{B}\left(\Lambda_{c}^{+} \rightarrow \Sigma^{-} \pi^{+} \pi^{+}\right)$needs an additional prefactor of $1 / 2$ to $T\left(\Lambda_{c}^{+} \rightarrow \Sigma^{-} \pi^{+} \pi^{+}\right)$due to the fact that the $\pi^{+} \pi^{+}$meson pair involves two identical bosons.
From $\mathcal{B}\left(\Lambda_{c}^{+} \rightarrow p K^{+} \pi^{-}\right) / \mathcal{B}\left(\Lambda_{c}^{+} \rightarrow p K^{-} \pi^{+}\right)=\tan ^{4} \theta_{c}$, we find that $\mathcal{R}_{K \pi}=1.1 \pm 0.3$ in the fit without the resonant part. The ratio of $\mathcal{R}_{K \pi} \sim 1$ is related to the same topological diagrams. Note that the experimental data of $\mathcal{R}_{K \pi}^{\text {Exp }}=0.58 \pm 0.06$ by LHCb [9] has been obtained by including the resonant contributions in $\Lambda_{c}^{+} \rightarrow p K^{-} \pi^{+}$. The predictions from the lowest-wave contributions, $\quad \mathcal{B}\left(\Lambda_{c}^{+} \rightarrow n \pi^{+} \bar{K}^{0}, p \bar{K}^{0} \pi^{0}\right)=(0.9 \pm 0.8$, $2.8 \pm 0.6) \times 10^{-2}$, are smaller than the data of $(3.6 \pm$ $0.6,4.0 \pm 0.3) \times 10^{-2}[3,5]$, which indicates that the resonant and/or high-wave contributions have not been clearly identified yet.

There exist the sum rules for the T-amplitudes in Table I. In particular, by taking the $\mathrm{CF} \Lambda_{c}^{+}$decay modes as an example, we obtain

$$
\begin{array}{r}
R(\Delta) \equiv T\left(\Lambda_{c}^{+} \rightarrow n \bar{K}^{0} \pi^{+}\right)-T\left(\Lambda_{c}^{+} \rightarrow p K^{-} \pi^{+}\right)-\sqrt{2} T\left(\Lambda_{c}^{+} \rightarrow p \bar{K}^{0} \pi^{0}\right)=0 \\
T\left(\Lambda_{c}^{+} \rightarrow \Sigma^{+} \pi^{0} \pi^{0}\right)-T\left(\Lambda_{c}^{+} \rightarrow \Sigma^{+} \pi^{+} \pi^{-}\right)+\frac{1}{2} T\left(\Lambda_{c}^{+} \rightarrow \Sigma^{-} \pi^{+} \pi^{+}\right)=0 \\
T\left(\Lambda_{c}^{+} \rightarrow \Sigma^{+} K^{0} \bar{K}^{0}\right)-T\left(\Lambda_{c}^{+} \rightarrow \Sigma^{+} K^{+} K^{-}\right)-\sqrt{2} T\left(\Lambda_{c}^{+} \rightarrow \Sigma^{0} K^{+} \bar{K}^{0}\right)=0 \\
T\left(\Lambda_{c}^{+} \rightarrow \Xi^{0} \pi^{+} K^{0}\right)-T\left(\Lambda_{c}^{+} \rightarrow \Xi^{-} \pi^{+} K^{+}\right)-\sqrt{2} T\left(\Lambda_{c}^{+} \rightarrow \Xi^{0} \pi^{0} K^{+}\right)=0 .
\end{array}
$$

Note that the first relation of $\mathcal{R}(\Delta)$ in Eq. (12), which has been used in Ref. [5] to reveal the broken isospin symmetry, is also derived by the isospin symmetry in Refs. [6,7] with some different signs in the relation due to the conventions of the $\pi^{+}$and $\bar{K}^{0}$ states. In addition, the second relation in Eq. (12) can be identified as the special case in Ref. [7], given by

$$
\begin{aligned}
& T\left(\Lambda_{c}^{+} \rightarrow \Sigma^{+} \pi^{0} \pi^{0}\right)-T_{\mathrm{sym}}\left(\Lambda_{c}^{+} \rightarrow \Sigma^{+} \pi^{+} \pi^{-}\right) \\
& \quad+\frac{1}{2} T\left(\Lambda_{c}^{+} \rightarrow \Sigma^{-} \pi^{+} \pi^{+}\right)=0
\end{aligned}
$$

with the symmetrized amplitude of

$$
\begin{aligned}
& T_{\text {sym }}\left(\Lambda_{c}^{+} \rightarrow \Sigma^{+} \pi^{+} \pi^{-}\right) \\
& \quad=\frac{1}{2}\left[T^{\prime}\left(\Lambda_{c}^{+} \rightarrow \Sigma^{+} \pi^{+} \pi^{-}\right)+T^{\prime}\left(\Lambda_{c}^{+} \rightarrow \Sigma^{+} \pi^{-} \pi^{+}\right)\right],
\end{aligned}
$$

where $T^{\prime}\left(\Lambda_{c}^{+} \rightarrow \Sigma^{+} \pi^{ \pm} \pi^{\mp}\right)$ are the amplitudes calculated

\begin{tabular}{|c|c|}
\hline CF mode & Our result \\
\hline $\begin{array}{l}10^{2} \mathcal{B}_{\Sigma^{+} \pi^{0} K^{-}} \\
10^{1} \mathcal{B}_{\Sigma^{+} \pi^{-} \bar{K}^{0}} \\
10^{3} \mathcal{B}_{\Sigma^{+} K^{-} \eta^{0}} \\
10^{2} \mathcal{B}_{\Sigma^{0} \pi^{0} \bar{K}^{0}} \\
10^{2} \mathcal{B}_{\Sigma^{0} \pi^{+} K^{-}} \\
10^{3} \mathcal{B}_{\Sigma^{0} \bar{K}^{0} \eta^{0}} \\
10^{2} \mathcal{B}_{\Xi^{0} \pi^{0} \pi^{0}} \\
10^{2} \mathcal{B}_{\Xi^{0} \pi^{0} \eta^{0}} \\
10^{1} \mathcal{B}_{\Xi^{0} \pi^{+} \pi^{-}} \\
10^{3} \mathcal{B}_{\Xi^{0} K^{+} K^{-}} \\
10^{4} \mathcal{B}_{\Xi^{0} \eta^{0} \eta^{0}} \\
10^{3} \mathcal{B}_{\Xi^{-} \pi^{0} \pi^{+}} \\
10^{2} \mathcal{B}_{\Xi^{-} \pi^{+} \eta^{0}} \\
10^{2} \mathcal{B}_{p K^{-} \bar{K}^{0}} \\
10^{3} \mathcal{B}_{n \bar{K}^{0} \bar{K}^{0}} \\
10^{2} \mathcal{B}_{\Lambda^{0} \pi^{0} \bar{K}^{0}} \\
10^{2} \mathcal{B}_{\Lambda^{0} \pi^{+} K^{-}}\end{array}$ & $\begin{array}{l}8.8 \pm 1.5 \\
1.8 \pm 0.3 \\
5.2 \pm 0.9 \\
4.4 \pm 1.1 \\
5.4 \pm 1.2 \\
1.4 \pm 0.3 \\
8.1 \pm 1.9 \\
1.2 \pm 0.2 \\
1.3 \pm 0.3 \\
3.6 \pm 0.9 \\
2.2 \pm 0.9 \\
4.6 \pm 1.2 \\
1.1 \pm 0.1 \\
1.2 \pm 0.1 \\
6.4 \pm 6.3 \\
2.0 \pm 0.6 \\
5.9 \pm 0.8\end{array}$ \\
\hline CS mode & Our result \\
\hline $\begin{array}{l}10^{4} \mathcal{B}_{\Sigma^{+} \pi^{0} \pi^{-}} \\
10^{3} \mathcal{B}_{\Sigma^{+} \pi^{-} \eta^{0}} \\
10^{3} \mathcal{B}_{\Sigma^{+} K^{0} K^{-}} \\
10^{3} \mathcal{B}_{\Sigma^{0} \pi^{0} \pi^{0}}\end{array}$ & $\begin{array}{l}7.2 \pm 0.7 \\
5.7 \pm 0.9 \\
2.4 \pm 0.4 \\
1.3 \pm 0.3\end{array}$ \\
\hline
\end{tabular}
by the isospin analysis in Ref. [7]. Likewise, one can take the relations in Eq. (12) to explore the broken $S U(3)_{f}$ symmetry. There are other relations and sum rules obtained from the U-spin symmetry, which is also a subgroup of $S U(3)_{f}[41] .^{2}$

\footnotetext{
${ }^{2}$ There is also a sign issue for the U-spin quantum state in Ref. [41].
}

TABLE VIII. Numerical results for the branching ratios of $\Xi_{c}^{0} \rightarrow \mathbf{B}_{\mathbf{n}} M M^{\prime}$, where $\mathcal{B}_{\mathbf{B}_{\mathbf{n}} M M^{\prime}} \equiv \mathcal{B}\left(\Xi_{c}^{0} \rightarrow \mathbf{B}_{\mathbf{n}} M M^{\prime}\right)$.

(Table continued) 
TABLE VIII. (Continued)

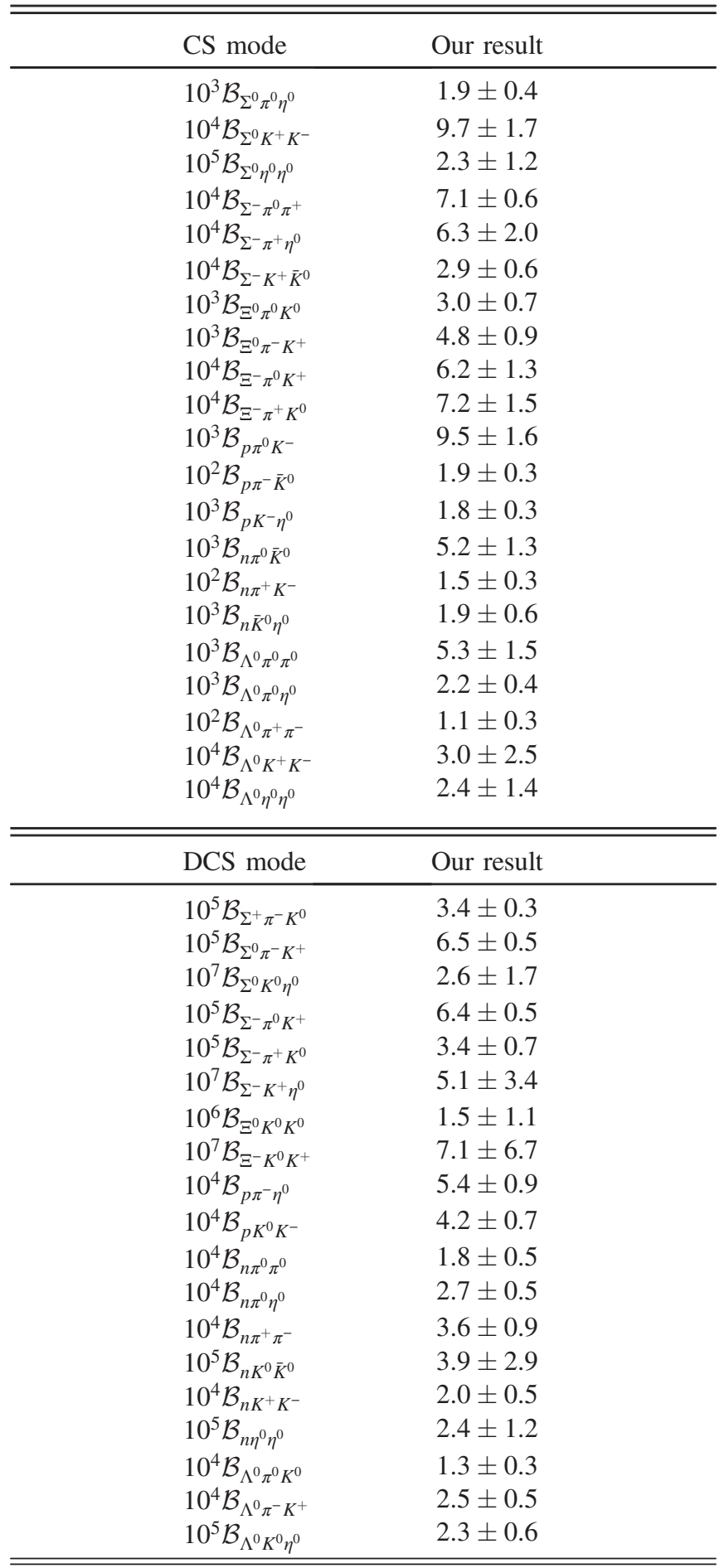

The not-yet-observed $\mathcal{B}\left(\Lambda_{c}^{+} \rightarrow \mathbf{B}_{\mathbf{n}} M M^{\prime}\right)$ can be calculated by the $S U(3)_{f}$ parameters, which are given in Table VI. The branching ratios of the three-body $\Xi_{c}^{+, 0}$ decays are partially observed, such that we predict $\mathcal{B}\left(\Xi_{c}^{+, 0} \rightarrow \mathbf{B}_{\mathbf{n}} M M^{\prime}\right)$ in Tables VII and VIII, respectively, to be compared to the upcoming data.

\section{CONCLUSIONS}

We have studied the three-body antitriplet $\mathbf{B}_{\mathbf{c}} \rightarrow$ $\mathbf{B}_{\mathbf{n}} M M^{\prime}$ decays in the approach of the $S U(3)_{f}$ symmetry. In our analysis, we have only concentrated on the S-wave $M M^{\prime}$-pair contributions, so that the decays of $\mathbf{B}_{\mathbf{c}} \rightarrow$ $\mathbf{B}_{\mathbf{n}} M M^{\prime}$ can be decomposed into irreducible forms with 11 parameters under $S U(3)_{f}$. With the minimum $\chi^{2}$ fit to the 14 existing data points, we have obtained a reasonable value of $\chi^{2} /$ d.o.f. $=2.8$. With our numerical results, we have shown the same triangle relation of $\mathcal{A}\left(\Lambda_{c}^{+} \rightarrow\right.$ $\left.n \bar{K}^{0} \pi^{+}\right)-\mathcal{A}\left(\Lambda_{c}^{+} \rightarrow p K^{-} \pi^{+}\right)-\sqrt{2} \mathcal{A}\left(\Lambda_{c}^{+} \rightarrow p \bar{K}^{0} \pi^{0}\right)=0$ under $S U(3)_{f}$ as that based on the isospin symmetry. In addition, for the $\mathrm{CF}$ decays, we have obtained the sum rules of $\mathcal{A}\left(\Lambda_{c}^{+} \rightarrow \Sigma^{+} \pi^{0} \pi^{0}\right)-\mathcal{A}\left(\Lambda_{c}^{+} \rightarrow \Sigma^{+} \pi^{+} \pi^{-}\right)+$ $1 / 2 \mathcal{A}\left(\Lambda_{c}^{+} \rightarrow \Sigma^{-} \pi^{+} \pi^{+}\right)=0, \quad \mathcal{A}\left(\Lambda_{c}^{+} \rightarrow \Sigma^{+} K^{0} \bar{K}^{0}\right)-$ $\mathcal{A}\left(\Lambda_{c}^{+} \rightarrow \Sigma^{+} K^{+} K^{-}\right)-\sqrt{2} \mathcal{A}\left(\Lambda_{c}^{+} \rightarrow \Sigma^{0} K^{+} \bar{K}^{0}\right)=0$, and $\mathcal{A}\left(\Lambda_{c}^{+} \rightarrow \Xi^{0} \pi^{+} K^{0}\right)-\mathcal{A}\left(\Lambda_{c}^{+} \rightarrow \Xi^{-} \pi^{+} K^{+}\right)-\sqrt{2} \mathcal{A}\left(\Lambda_{c}^{+} \rightarrow\right.$ $\left.\Xi^{0} \pi^{0} K^{+}\right)=0$. Furthermore, we have predicted that $\mathcal{B}\left(\Lambda_{c}^{+} \rightarrow n \pi^{+} \bar{K}^{0}\right)=(0.9 \pm 0.8) \times 10^{-2}$, which is 3-4 times smaller than the BESIII observation of $(3.6 \pm 0.6) \times$ $10^{-2}$. This indicates that there are some contributions from the resonant and/or P-wave states. For the to-be-observed $\Lambda_{c}^{+} \rightarrow \mathbf{B}_{\mathbf{n}} M M^{\prime}$ and the partially observed $\Xi_{c}^{0,+} \rightarrow \mathbf{B}_{\mathbf{n}} M M^{\prime}$ decays, the branching ratios have been calculated with the $S U(3)_{f}$ amplitudes, to be compared to the future measurements by BESIII and LHCb.

\section{ACKNOWLEDGMENTS}

This work was supported in part by the National Center for Theoretical Sciences, Ministry of Science and Technology (MoST) (No. MoST-104-2112-M-007-003MY3 and No. MoST-107-2119-M-007-013-MY3), and the National Science Foundation of China (11675030). 
[1] A. Zupanc et al. (Belle Collaboration), Phys. Rev. Lett. 113, 042002 (2014).

[2] M. Ablikim et al. (BESIII Collaboration), Phys. Rev. Lett. 116, 052001 (2016).

[3] C. Patrignani et al. (Particle Data Group), Chin. Phys. C 40, 100001 (2016).

[4] M. Berger et al. (Belle Collaboration), Phys. Rev. D 98, 112006 (2018).

[5] M. Ablikim et al. (BESIII Collaboration), Phys. Rev. Lett. 118, 112001 (2017).

[6] C. D. Lu, W. Wang, and F. S. Yu, Phys. Rev. D 93, 056008 (2016).

[7] M. Gronau, J. L. Rosner, and C. G. Wohl, Phys. Rev. D 97, 116015 (2018); 98, 073003(A) (2018).

[8] S. B. Yang et al. (Belle Collaboration), Phys. Rev. Lett. 117, 011801 (2016).

[9] R. Aaij et al. (LHCb Collaboration), J. High Energy Phys. 03 (2018) 043.

[10] J. D. Bjorken, Phys. Rev. D 40, 1513 (1989).

[11] A. Ali, G. Kramer, and C. D. Lu, Phys. Rev. D 58, 094009 (1998).

[12] C. Q. Geng, Y. K. Hsiao, and J. N. Ng, Phys. Rev. Lett. 98, 011801 (2007).

[13] Y. K. Hsiao and C. Q. Geng, Phys. Rev. D 91, 116007 (2015).

[14] H. Y. Cheng and B. Tseng, Phys. Rev. D 46, 1042 (1992); 55, 1697(E) (1997).

[15] H. Y. Cheng and B. Tseng, Phys. Rev. D 48, 4188 (1993).

[16] P. Zenczykowski, Phys. Rev. D 50, 402 (1994).

[17] Fayyazuddin and Riazuddin, Phys. Rev. D 55, 255; 56, 531(E) (1997).

[18] R. Dhir and C. S. Kim, Phys. Rev. D 91, 114008 (2015).

[19] H. Y. Cheng, X. W. Kang, and F. Xu, Phys. Rev. D 97, 074028 (2018).

[20] X. G. He, Y. K. Hsiao, J. Q. Shi, Y. L. Wu, and Y. F. Zhou, Phys. Rev. D 64, 034002 (2001).

[21] H. K. Fu, X. G. He, and Y. K. Hsiao, Phys. Rev. D 69, 074002 (2004).
[22] Y. K. Hsiao, C. F. Chang, and X. G. He, Phys. Rev. D 93, 114002 (2016).

[23] X. G. He and G. N. Li, Phys. Lett. B 750, 82 (2015).

[24] M. He, X. G. He, and G. N. Li, Phys. Rev. D 92, 036010 (2015).

[25] Y. Grossman and D. J. Robinson, J. High Energy Phys. 04 (2013) 067.

[26] D. Pirtskhalava and P. Uttayarat, Phys. Lett. B 712, 81 (2012).

[27] H. Y. Cheng and C. W. Chiang, Phys. Rev. D 86, 014014 (2012).

[28] M. J. Savage and R. P. Springer, Phys. Rev. D 42, 1527 (1990).

[29] M. J. Savage, Phys. Lett. B 257, 414 (1991).

[30] G. Altarelli, N. Cabibbo, and L. Maiani, Phys. Lett. 57B, 277 (1975).

[31] C. Q. Geng, Y. K. Hsiao, Y. H. Lin, and L. L. Liu, Phys. Lett. B 776, 265 (2018).

[32] C. Q. Geng, Y. K. Hsiao, C. W. Liu, and T. H. Tsai, Phys. Rev. D 97, 073006 (2018).

[33] C. Q. Geng, Y. K. Hsiao, C. W. Liu, and T. H. Tsai, J. High Energy Phys. 11 (2017) 147.

[34] C. Q. Geng, Y. K. Hsiao, C. W. Liu, and T. H. Tsai, Eur. Phys. J. C 78, 593 (2018).

[35] W. Wang, Z. P. Xing, and J. Xu, Eur. Phys. J. C 77, 800 (2017).

[36] D. Wang, P. F. Guo, W. H. Long, and F. S. Yu, J. High Energy Phys. 03 (2018) 066.

[37] C. Q. Geng, C. W. Liu, and T. H. Tsai, Phys. Lett. B 790, 225 (2019).

[38] A. J. Buras, arXiv:hep-ph/9806471.

[39] H. n. Li, C. D. Lu, and F. S. Yu, Phys. Rev. D 86, 036012 (2012).

[40] S. Fajfer, P. Singer, and J. Zupan, Eur. Phys. J. C 27, 201 (2003).

[41] Y. Grossman and S. Schacht, Phys. Rev. D 99, 033005 (2019). 